\title{
MECHANICAL CHARACTERIZATION OF ADDITIVELY MANUFACTURED MICROSTRUCTURES USING A PROCESS INTEGRATED MEMS TENSILE TESTER
}

Ian S. Ladner ${ }^{1,2+}$, Joon H. Cho ${ }^{2}$, David R. Cayll ${ }^{2}$, Vu H. Nguyen ${ }^{l}$, Michael A. Cullinan ${ }^{2 *}$, and Sourabh K. Saha ${ }^{1 *}$

${ }^{1}$ Lawrence Livermore National Laboratory, USA

${ }^{2}$ University of Texas at Austin, USA

\begin{abstract}
With the rapid growth of additive manufacturing technologies, mechanical characterization of printed structures is becoming increasingly important to ensure their suitability as functional components. However, characterization of microstructures is traditionally compression testing due to handling challenges of at-scale parts. To overcome that limitation, we have designed, fabricated, and tested custom microelectromechanical system (MEMS) tensile tester that enables direct integration with additively manufactured (AM) parts with $\mathrm{nm}$ displacement resolution and $\mathrm{mN}$ force range. This work characterizes the mechanical behavior of two AM parts stitched together. At yield, the failure strain is $3.1 \%$, which is $3 \mathrm{x}$ lower than under compression.
\end{abstract}

\section{INTRODUCTION}

Miniaturization of mechanical testing instruments through use of microelectromechanical systems (MEMS) has been successfully performed in the past to characterize small-scale structures [1]. Unfortunately, indirect integration through pickand-place techniques is not appropriate for characterization of additively manufactured (AM) polymeric parts, which are easilydeformed. Additionally, existing MEMS tensile testers fail to maintain their functionality after direct integration of AM parts onto the sensors. To overcome these limitations, we have designed, built, and tested custom MEMS tensile testers that enable direct integration of AM parts (Fig. 1). Suitability for AM process integration was ensured through geometric design that minimizes stiction effects. Preliminary design of the tensile testers was presented at the ASPE 2017 annual meeting [2]. Here, we present the device fabrication, sensor calibration, and mechanical testing data (stress-strain curve) generated from the MEMS testers with integrated AM parts.

We have used two-photon lithography (TPL) to fabricate test structures in this work. TPL is a laser-based polymerization technique that enables fabrication of sub-diffraction features that are $100-500 \mathrm{~nm}$ wide and smaller than the focused light spot
(Fig. 2). Sub-diffraction resolution is ensured by the underlying non-linear two-photon absorption process that occurs at the high light intensities $\left(\sim \mathrm{TW} / \mathrm{cm}^{2}\right)$ achieved through focusing of femtosecond lasers. Microstructures are generated by scanning the focused light spot in 3D space. Due to the limited range of scanners, larger structures are generated by "stitching" multiple sections. These stitch lines often act as regions of reduced strength due to alignment errors and need to be characterized to enable deterministic design of millimeter-scale parts.

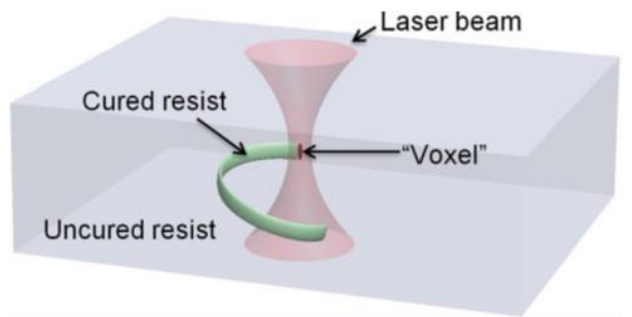

Figure 2: Schematic of two-photon lithography illustrating sub-diffraction printing [3].

\section{DESIGN}

The tensile tester is comprised of a chevron style thermal actuator and two differential capacitive sensors for load and displacement sensing (Fig. 1.a). A thermal actuator was chosen to achieve the high force and relatively large displacement required to achieve $>2 \%$ strain on the AM microstructures. Single-sided differential capacitors were selected to generate a high selectivity for a given footprint to achieve nm displacement resolution (Fig. 1.c). A twosensor design was selected to simultaneously measure stress and strain without requiring an additional vision-based metrology system. The actuator and displacement sensor share the same moving shuttle that is guided by a flexure bearing whereas the load sensor is guided by a separate flexure bearing. The tip of the displacement sensor and the load sensor is the print location for the AM part with the gap. For dimensions and details on design method see [2].

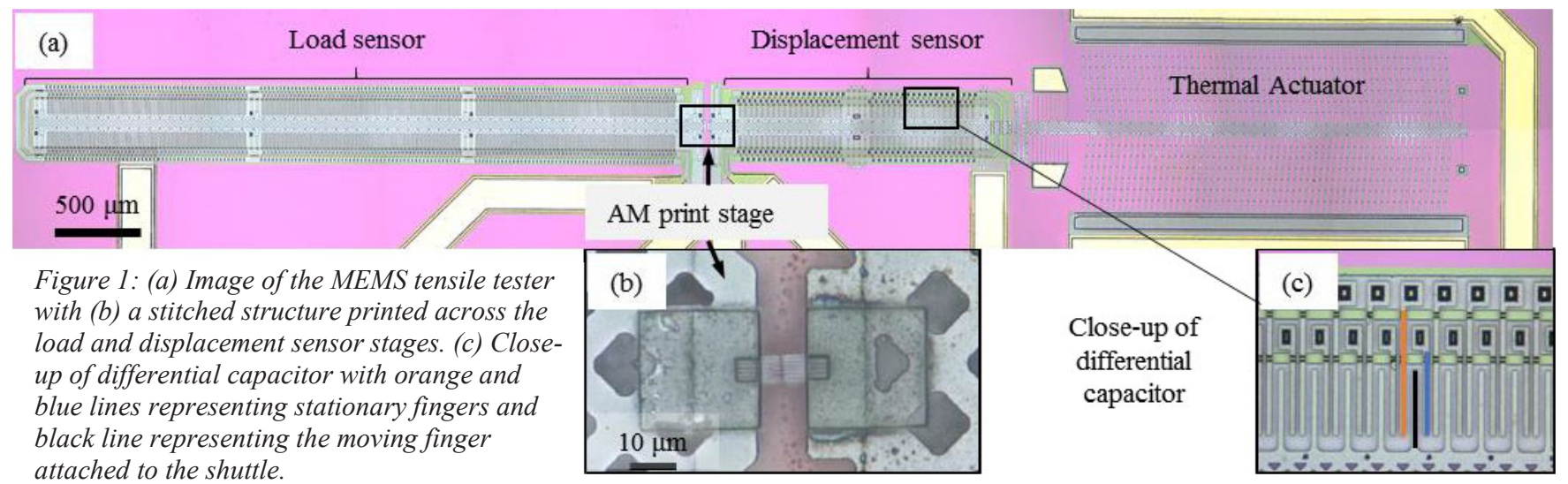




\section{FABRICATION}

A two-layer PolyMUMPs process was used for this work, which is required for the single-sided differential capacitors. A simplified process flow is shown in Fig. 3. A $600 \mathrm{~nm}$ silicon nitride layer was deposited as an electrical insulator between the tester and a heavily doped silicon substrate. One micron of amorphous silicon was deposited and doped with a spin-on phosphosilicate glass (PSG) dopant. An anneal was run at $1050^{\circ} \mathrm{C}$ to dope and crystallize the layer into polysilicon. The Polysilicon 1 layer was patterned to form the electrical network between components. A $1 \mu \mathrm{m}$ layer of thermal oxide, Oxide 1, was deposited to planarize the area under the thermal actuator. The second thermal oxide layer, Oxide 2, was deposited to create the $2.5 \mu \mathrm{m}$ air gap under the device layer and patterned with holes to connect the device layer to Polysilicon 1 . Next, the $8 \mu \mathrm{m}$ device layer, Polysilicon 2, was deposited and doped with PSG. Prior to patterning the device layer, $300 \mathrm{~nm}$ of gold was deposited with a bi-layer lift-off to form electrical traces and bond pads. Polysilicon 2 was pattern with deep reactive ion etching (DRIE) to define the thermal actuator and capacitive sensor features. After dicing, a buffered oxide etch (BOE) and deionized water (DI) solution was used to remove the two thermal oxide layers and release the suspended portions of the Polysilicon 2 layer. Chips were transferred into DI followed by isopropyl alcohol (IPA) and drying in air to complete the release process. The chips were wire bonded to a custom PCB package, which was designed for integration into the Nanoscribe Photonic Professional (GT) $3 \mathrm{D}$ printing system.

(a)

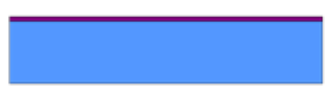

(c)

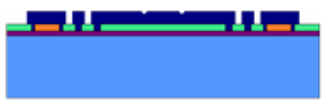

(e)

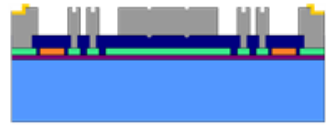

Silicon

Oxide $2 \square$ Polysilicon 2

Nitride

(b)

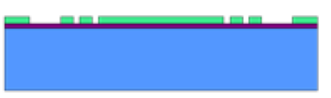

(d)

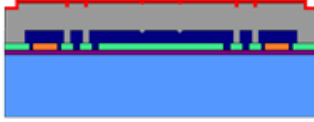

(f)

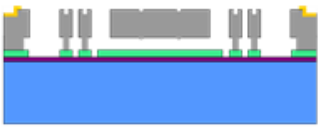

Polysilicon 1

Oxide 1

PSG

$\mathrm{Au}$

Figure 3: Fabrication process flow

\section{CALIBRATION}

Thermal actuator was calibrated to generate a displacement versus input voltage curve. Digital image correlation (DIC) was used to measure the displacement of moving finger on the shuttle (black finger in Fig. 1.c) with respect to the pair of stationary fingers (orange and blue fingers in Fig. 1.c) at each thermal actuator voltage. Fig. 4.a shows the calibration curve, whose nonlinear response at higher voltage is as predicted [2].

With the displacement sensor connected to the shuttle of the thermal actuator, the change in capacitance for a given displacement was measured at the same time. The change in the capacitance, $\Delta C$, of the sensor was calculated from the voltage response of the Irvine Sensors MS3110IC capacitive readout. Fig. 4.b shows the $\Delta C$ versus displacement calibration curves for the displacement sensor. The load sensor calibration was generated during the initial portion of the tensile test. The
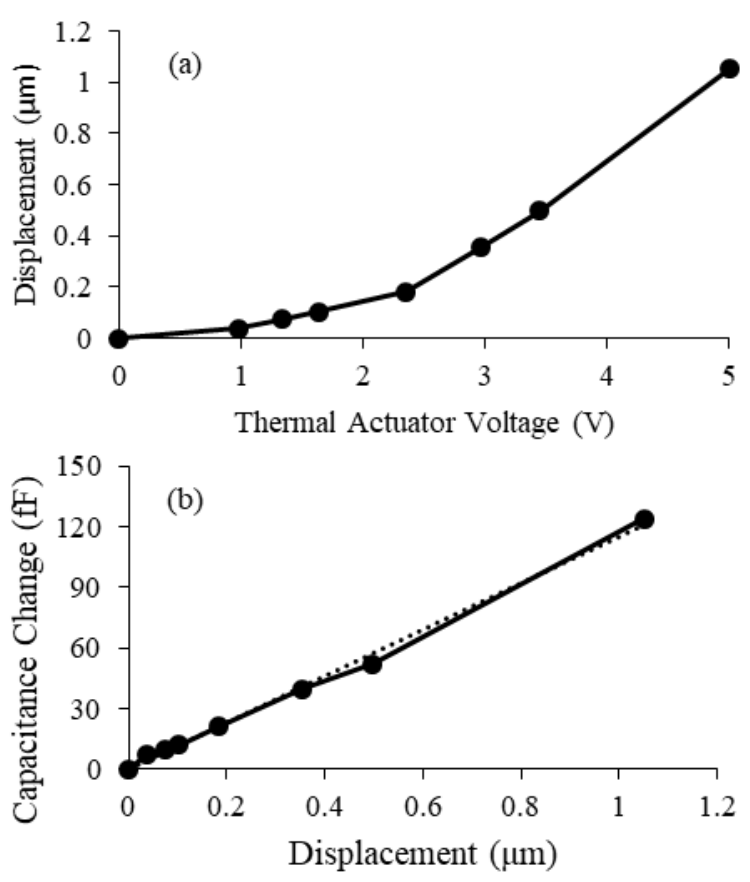

Figure 4: (a) Displacement vs. thermal actuator voltage, and (b) $\Delta C v$ s. displacement calibration curve for the displacement sensor

fabricated displacement sensor has a calibrated resolution of $3 \mathrm{~nm}$ and an operating range of $1.05 \mu \mathrm{m}$.

\section{RESULTS AND DISCUSSION}

A stitched part was printed directly on top of the MEMS tester with writing power of $20 \mathrm{~mW}$ and speed at $10 \mathrm{~mm} / \mathrm{s}$ (Fig. 1.b) using the AM integration summarized in Fig. 5. A droplet of photoresist was placed on the tester and loaded into the Nanoscribe Photonic Professional (GT). The galvanometer
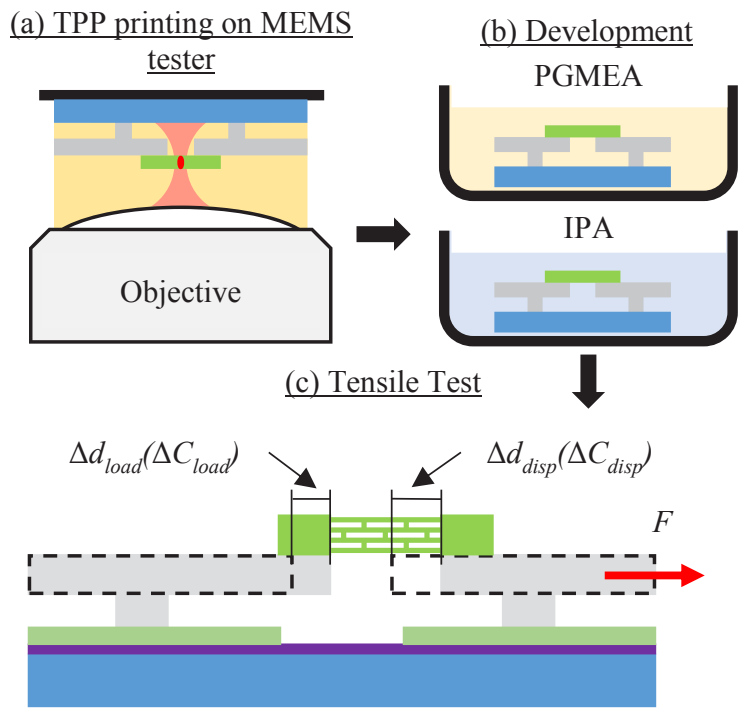

Figure 5: Schematic of AM + MEMS process flow: (a) TPL printing directly on MEMS tester, (b) PGMEA and IPA to develop and clean, and (c) tensile testing after drying. 
scanner wrote the microstructure between the shuttle ends of each sensor. The un-patterned resist was removed by propylene glycol monomethyl ether acetate (PGMEA), and the device was cleaned in IPA. After air drying, the device was connected to the electronics and ready for tensile testing.

A schematic of the stitched region of the structure is shown in Fig. 6. The voxel lines were printed $10 \mu \mathrm{m}$ long in $\mathrm{X}$ and $6 \mu \mathrm{m}$ in $\mathrm{Y}$ with a cross section of approximately $350 \mathrm{~nm}$ in diameter, $d$, and $1.25 \mu \mathrm{m}$ in height. The pitch, $P$, in the top view was $500 \mathrm{~nm}$ in both direction. At the stitch, the offsets were $\delta x=375 \mathrm{~nm}$ and $\delta \mathrm{Y}=250 \mathrm{~nm}$ between the red and blue structures. In the cross section view, the vertical offset was $500 \mathrm{~nm}$ for each of the 5 layers. The gauge length for the printed structure was $10.02 \mu \mathrm{m}$ with a final thickness of $2.75 \mu \mathrm{m}$.

For testing the stitched structure, a dc voltage was applied to the thermal actuator while output signal from the MS3110IC for displacement and load sensors were captured. Deformation of the stitch part was recorded optically. The voltage was increased through the full range of the thermal actuator. Displacement measured from the load sensor was converted into force using the known stiffness of $12.9 \mathrm{kN} / \mathrm{m}$ for the flexure bearings [2] with a resolution of $38.7 \mu \mathrm{N}$ and range of $0.3 \mathrm{mN}$.

The engineering stress-strain curve is shown in Fig. 7. The measured Young's modulus of the stitched part was $1.45 \mathrm{GPa}$. At yield, necking was observed at the stitched region shown in Fig. 7.b. The failure strain was $3.1 \%$ which is significantly lower than the failure strain in compression $(>10 \%)$.

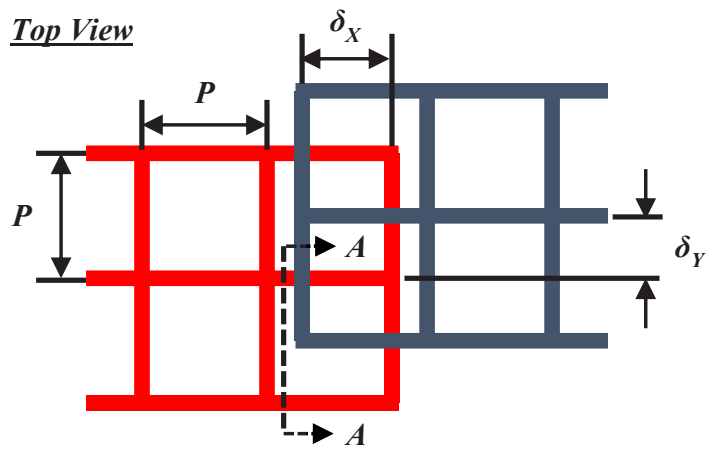

Cross section View $A-A$

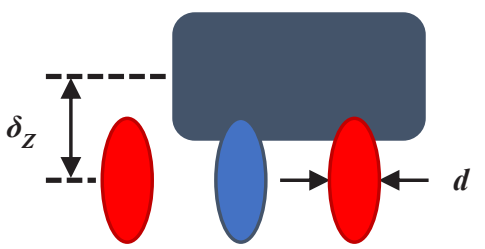

Figure 6: Top view of the stitched structure with pitch, $P$, and in-plane offsets, $\delta_{X}$ and $\delta_{Y}$, and cross section view with the voxel width, $d$, and $z$ spacing, $\delta_{Z}$, between the lateral and longitudinal layers.

\section{CONCLUSION}

The mechanical properties of a TPL stitched AM part were quantified by printing directly onto a custom MEMS tensile tester. The measured properties show a lower yield threshold when compared to the traditional compression testing methods demonstrating the value of direct tensile testing. The process integration demonstrated in this work will lead to mechanical characterization of scale-dependent phenomena, well-

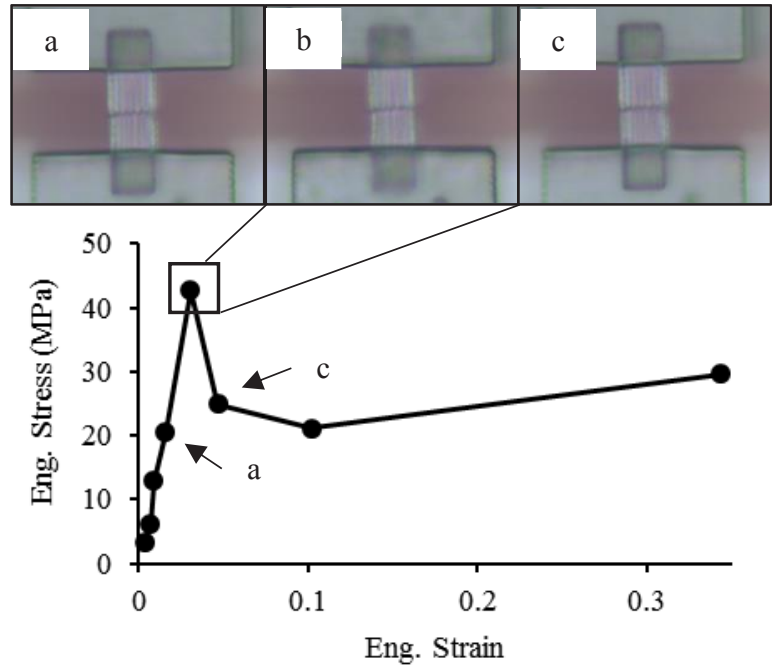

Figure 7: Eng. stress vs. Eng. strain curve with images before (a), during (b), and after yielding (c). Young's modulus measured at $1.45 \mathrm{GPa}$.

characterized custom resists, and more accurate quantification of the effect of process parameters on material properties. These are critical to ensure growth of TPL into a robust manufacturing technique. Additionally, these sensors open the door to study other liquid transferable nano- to microscale materials which require scale-dependent testing with minimal impact due to sample handling.

\section{ACKNOWLEDGEMENTS}

This work was performed under the auspices of the U.S. Department of Energy by Lawrence Livermore National Laboratory under Contract DE-AC52-07NA27344 and by the University of Texas at Austin under subcontracts B617787, B621043, B626195. Funding available from LDRD-16-ERD047. LLNL-PROC-748753. The authors thank the staff at the Microelectronics Research Center of University of Texas at Austin for assistance during fabrication, Marianne Ammendolia (LLNL) for packaging of chips, and Steve Hunter (LLNL) for assistance in the design of the sensing electronics.

\section{REFERENCES:}

[1] Y. Zhu and T.H. Chang, "A review of microelectromechanical systems for nanoscale mechanical characterization," J. Micomech. Microeng., vol. 25, no. 9, 2015.

[2] I.S. Ladner et. al., "Design of high resolution and high force MEMS tensile tester for direct metrology of additive manufactured submicron features, " in $32^{\text {nd }}$ Annual American Society of Precision Engineering, Charlotte, NC, 2017.

[3] S.K. Saha et. al., "Effect of Proximity of Features on the Damage Threshold during Submicron Additive Manufacturing Via Two-Photon Polymerization, " J. Micro Nano-Manufacturing, vol. 5, no. 3, 2017.

\section{Contact:}

†. S. Ladner, tel: 1-925-424-4260; ladner2@1lnl.gov

*Corresponding authors: michael.cullinan@austin.utexas.edu, saha5@1lnl.gov 